\title{
True or false - the Irish consumer's attitude to nutrition and health claims
}

\author{
C.M. Donovan ${ }^{1}$, C.B. O’Donovan ${ }^{1}$, S. O'Mahony ${ }^{1,2}$, O.C. Lyons ${ }^{1,3}$, M.A.T. Flynn ${ }^{1,3}$ and \\ N.A. Collins 1 \\ ${ }^{1}$ Food Safety Authority of Ireland, Dublin 1, Republic of Ireland, \\ ${ }^{2}$ University College Dublin, Dublin 4, Republic of Ireland and \\ ${ }^{3}$ Nutrition Innovation Centre for Food and Health, Ulster University, Coleraine, BT52 ISA, UK
}

Nutrition and health claims on food is legislated for under Commission Regulation (EC) 1924/2006 ${ }^{(1)}$ and Statutory Instrument No. 11 of $2014^{(2)}$. These pieces of legislation ensure that any claim made on a food product label is clear, accurate and substantiated, allowing consumers to make informed decisions. A recent systematic review has shown that claims on food labels influence purchasing intentions of European consumers ${ }^{(3)}$. In an Irish context, recent research investigating consumer attitudes towards nutrition and health claims is lacking. The aim of this study was to explore the attitudes of a representative sample of Irish consumers in relation to the use of nutrition and health claims. This work fits within a larger compliance building project being carried out by The Food Safety Authority of Ireland (FSAI) and Environmental Health Service (EHS) with respect to Commission Regulation (EC) 1924/2006.

The FSAI contracted Amárach Research, a market research and data specialist company, to undertake a quantitative omnibus survey in order to better understand consumer attitudes towards nutrition and health claims. An omnibus survey of $n 1000$ participants was undertaken according to quotas set by Amárach for gender, age, social class and geographical region to achieve a nationally representative sample. The survey comprised of 5 close-ended questions which was completed online by participants.

The survey revealed $78 \%$ of consumers trust the claims made on food labels. The participants also showed a high level of awareness of the presence of claims on food labelling with $97 \%$ self-reporting that they could identify nutrition and health claims, and $86 \%$ actively seeking out products which advertise claims. The data indicated that consumers were more heavily influenced by health claims compared to nutrition claims with $78 \%$ of participants stating that health claims influence purchases. However, this research showed that consumer knowledge was lacking in discerning true from untrue claims, as $83 \%$ incorrectly identified an unauthorised claim to be true. The unauthorised claim which consumers identified to be true was 'biotin is necessary for healthy teeth, bones, hair and skin', while $17 \%$ correctly identified the authorised claim 'biotin contributes to the maintenance of normal hair' to be true.

The findings of the omnibus survey provide an updated insight into consumer attitudes towards nutrition and health claims. Consumers cannot be expected to know or keep up to date with authorised or newly authorised claims and can easily be misled by untrue claims. The high level of trust in food labels reported by consumers makes it imperative to help food businesses comply with nutrition and health claims labeling legislation. The ongoing FSAI \& EHS compliance building programme aims to improve food businesses compliance with Commission Regulation 1924/2006 while also protecting consumers.

1. Regulation (EC) no.1924/2006 of the European Parliament and of the Council of 20 December 2006 on Nutrition and Health Claims Made on Foods

2. S.I. No.11/2014 - European Union (Nutrition and Health Claims made on Foods) Regulations 2014

3. Oostenbach LH, Slits E, Robinson E et al. (2019) BMC Public Health 19 